\title{
A Rare Case of Small Bowel Intussusception Due to Metastatic Malignant Melanoma
}

\author{
Yuma Hanaoka', Atsushi Tanemura ${ }^{*}$, Mari Tanaka1, Madoka Takafuji1, Eiji Kiyohara1, \\ Mari Wataya-Kaneda', Manabu Fujimoto', Naotsugu Haraguchi' ${ }^{2}$ \\ ${ }^{1}$ Department of Dermatology, Osaka University Graduate School of Medicine, Suita, Osaka, Japan \\ ${ }^{2}$ Department of Gastroenterological Surgery, Osaka University Graduate School of Medicine, Suita, Osaka, Japan \\ Email: *tanemura@derma.med.osaka-u.ac.jp
}

How to cite this paper: Hanaoka, Y., Tanemura, A., Tanaka, M., Takafuji, M., Kiyohara, E., Wataya-Kaneda, M., Fujimoto, M. and Haraguchi, N. (2019) A Rare Case of Small Bowel Intussusception Due to Metastatic Malignant Melanoma. Journal of Cosmetics, Dermatological Sciences and Applications, 9, 107-112.

https://doi.org/10.4236/jcdsa.2019.92009

Received: April 15, 2019

Accepted: May 26, 2019

Published: May 29, 2019

Copyright $\odot 2019$ by author(s) and Scientific Research Publishing Inc. This work is licensed under the Creative Commons Attribution International License (CC BY 4.0).

http://creativecommons.org/licenses/by/4.0/

\begin{abstract}
The small intestine is sometimes metastasized by malignant melanoma. While small intestinal involvement is asymptomatic or usually nonspecific, intussusception causing intestinal obstruction is very rare. Herein we report the case of a 68-year-old man with small bowel intussusception due to metastatic malignant melanoma. Although he has been comprehensively treated with surgery, radiation, and immunotherapies, the disease rapidly progressed and consequently severe life-threatening intussusception developed on the small bowel. Because surgical resection of the obstructive melanoma lesions was successful, his quality of life was dramatically improved followed by the resumption of treatment with anti-PD-1. Several effective treatments for metastatic melanoma, including immune checkpoint inhibitors and targeted therapy, have been developed within the past decade. However, given the present case, surgical approaches to symptomatic metastatic melanoma lesions should still be considered as a treatment option for improving not only patients' critical condition but also their survival.
\end{abstract}

\section{Keywords}

Intussusception, Bowel Obstruction, Metastatic Melanoma, Palliative Surgery, Sequential Anti-Tumor Immunotherapy

\section{Introduction}

The small intestine is sometimes metastasized by malignant melanoma [1] [2] [3]. Symptoms of small intestinal involvement are asymptomatic or usually nonspecific, like abdominal pain, nausea, and vomiting, which leads to a late diagnosis often made only after complications arise [4] [5] [6]. The most common complication is intestinal obstruction [6], and intussusception causing intestinal 
obstruction is very rare [7]. Because life is threatened once intussusception occurs, it often requires emergent surgical removal of obstructive lesions along with the neighboring bowel [8]. We herein report a rare case of metastatic melanoma causing small bowel intussusception.

\section{Case Presentation}

A 68-year-old man had been affected by acral lentiginous melanoma (pT4b) on the right heel and received wide local resection of his primary lesion and a sentinel lymph node biopsy. Because one popliteal lymph node was positive for micro-metastasis, he subsequently underwent right popliteal lymph node dissection (one metastatic node out of four). Because lymph node metastasis was suspected during treatment with adjuvant interferon beta therapy, we performed right inguinal lymph node dissection (no metastatic nodes out of 18). Ten months later, a solitary metastasis to the left tenth rib without $B R A F$ mutation was recognized by positron emission tomography (PET)-computed tomography (CT) (Figure 1 ), and surgical resection was performed.

Three months after surgery, multiple metastases to the stomach were detected, and due to rapid disease progression, he was treated with an anti-PD-1 antibody (pembrolizumab; $2 \mathrm{mg} / \mathrm{kg}$ every 3 weeks). After nine administrations of pembrolizumab, CT detected several new small intestinal metastases. The treatment was sequentially switched to anti-CTLA- 4 antibody (ipilimumab; $3 \mathrm{mg} / \mathrm{kg}$ every 3 weeks) concomitant with $30 \mathrm{~Gy} / 10 \mathrm{Fr}$ radiotherapy to stomach metastases, expecting an abscopal effect. After four administrations of ipilimumab without severe adverse events, including colitis, he was unable to receive a diet orally. $\mathrm{Ab}$ dominal radiography showed prominent loops of the small bowel and air fluid in the mid- and upper abdomen. Abdominal CT revealed a fully-matured mass and edema in the small bowel, suspected of being intussusception (Figure 2(a) and Figure 2(b)). Laparoscopic surgery was performed to alleviate the symptoms of intestinal obstruction as an emergency, and not only to prevent complications. During the operation, we observed small bowel intussusception with the lead point being an intraluminal intestinal mass (Figure 3(a) and Figure 3(b)) and another mass in the proximal ileum (Figure $3(\mathrm{c})$ ). Small bowel resection was performed with end-to-end anastomosis (Figures 3(b)-(d)). A histopathologic examination of the small bowel lesion confirmed metastatic melanoma (Figure 3(e) and Figure 3(f)). The post-operative period was uneventful, and the patient was discharged on the $12^{\text {th }}$ postoperative day. After discharge, he was able to consume an oral diet, and immunotherapy with the anti PD-1 antibody nivolumab was resumed. He ultimately died 5 months after resection of the small bowel lesions and 44 months after surgery for the primary lesion.

\section{Discussion}

Intussusception results from the invagination of the proximal segment of the bowel into the lumen of a distal segment and is known to be associated with an 


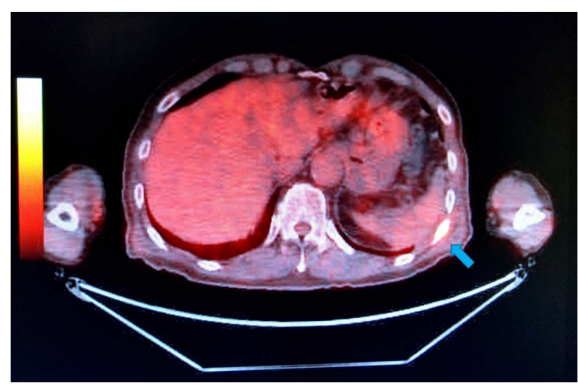

Figure 1. Positron emission tomography-computed tomography showing fluorodeoxyglucose avid at the left tenth rib (arrowed).
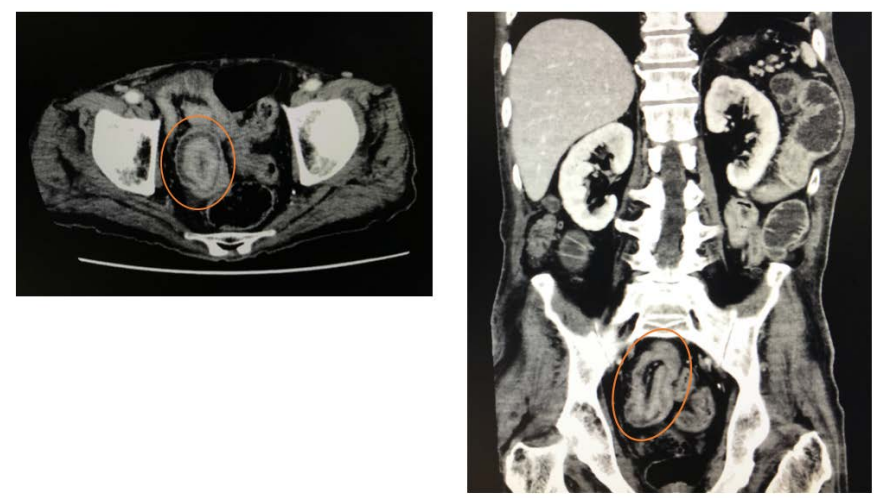

(a)

(b)

Figure 2. Preoperative computed tomography showing a small bowel mass causing intussusception (encircled). (a) and (b) indicate axial and coronal sections, respectively.

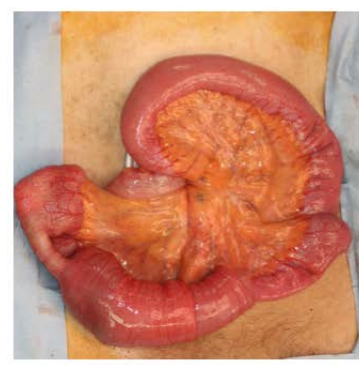

(a)

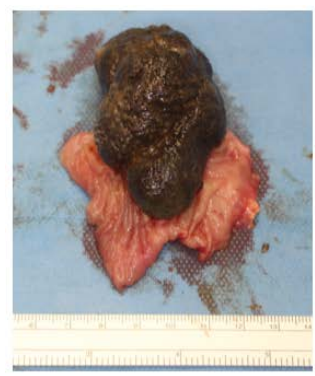

(d)

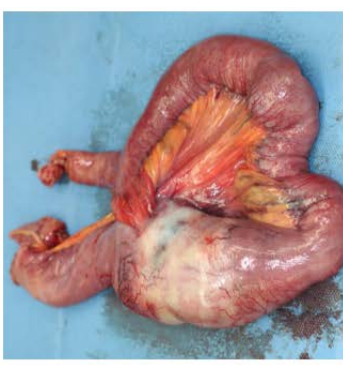

(b)

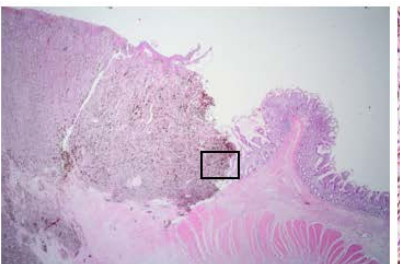

(e)
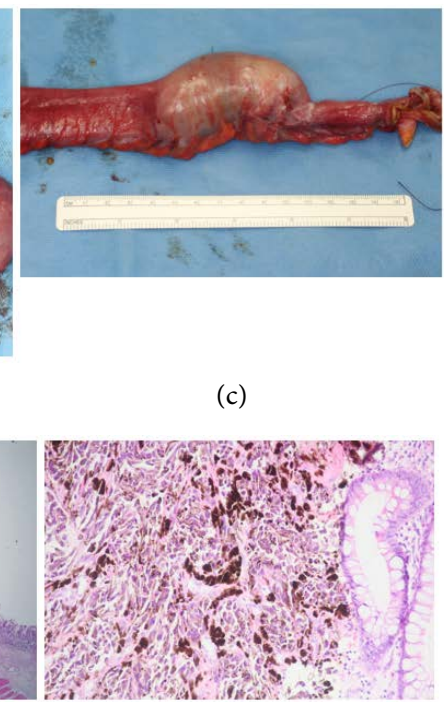

(c)

(f)

Figure 3. (a) Intraoperative image of the intussusception; (b) (c) Surgical specimen showing the invaginated bowel segment ((b) the distal ileum, (c) the proximal ileum); (d) Open surgical specimen revealing a $3 \times 7-\mathrm{cm}$ neoplasia; (e) (f) Histopathological findings showed the presence of melanoma cells next to the mucosal epithelia $((e) \times 12.5,(f) \times 100)$. 
underlying primary-not metastatic-malignant neoplasm as the leading point [9] [10]. Intussusception often requires emergent surgical removal of obstructive lesions along with the neighboring bowel [8]. While intestinal and mesenteric metastases of malignant melanoma are usually disseminated and small, intussusception related to intraluminal masses of melanoma is quite uncommon [8]. The usual time from the diagnosis of the primary tumor to that of metastases is usually within 2 - 5 years [11]; about $1.5 \%-4.4 \%$ of all patients with melanoma are diagnosed antemortem, while $50 \%-60 \%$ of patients with malignant melanoma present with metastases at an autopsy [1].

While immune checkpoint inhibitors (anti PD-1 antibody and/or anti CTLA-4 antibody) have provided unprecedented gains in metastatic or advanced melanoma treatment [12], the efficacy of immunotherapy on melanoma remains limited, and an increase in their anti-tumor efficacy is required urgently. Although the present case was comprehensively treated with surgery, radiation, and immunotherapies, the disease rapidly progressed, and severe intussusception of the small bowel was consequently detected by CT.

Because surgical resection of the obstructive melanoma lesions was successful, his quality of life was dramatically improved followed by the resumption of treatment with anti-PD-1. Several effective treatments for metastatic melanoma, including immune checkpoint inhibitors and targeted therapy, have been developed within the past decade [13]. However, given the present case, surgical approaches to symptomatic metastatic melanoma lesions should still be considered as a treatment option for improving not only patients' critical condition but also their survival.

\section{Conclusion}

The small intestine is sometimes metastasized by malignant melanoma while intussusception related to intraluminal masses of melanoma is quite uncommon. Although the evolving role of immunotherapy and targeted therapy of metastatic malignant melanoma has been extended survival, surgical approaches to symptomatic metastatic melanoma lesions should still be considered as a treatment option for improving not only patients' critical condition but also their survival.

\section{Acknowledgements}

We greatly appreciate Mr. Kenji Nishida for an excellent tissue staining.

\section{Consent}

Written informed consent was obtained from the patient's sister for publication of this case report.

\section{Statement of Ethics}

The authors have no ethical conflicts to disclose. 


\section{Disclosure Statement}

The authors declare that no competing interests exist.

\section{Funding Sources}

None of the authors received any financial support for the present study.

\section{Author Contributions}

All authors participated in patient care, obtaining patient history and workup, and writing the manuscript.

\section{References}

[1] Lens, M., Bataille, V. and Krivokapic, Z. (2009) Melanoma of the Small Intestine. The Lancet Oncology, 10, 516-521. https://doi.org/10.1016/s1470-2045(09)70036-1

[2] Kotteas, E.A., Adamopoulos, A., Drogitis, P.D., Zalonis, A., Giannopoulos, K.V., Karapanagiotou, E.M., Saif, M.W. and Syrigos, K.N. (2009) Gastrointestinal Bleeding as Initial Presentation of Melanoma of Unknown Primary Origin: Report of a Case and Review of the Literature. In Vivo, 23, 487-490.

[3] López, R., Santomé, P., Porto, E., Moreiras, M., Gómez, C., Villanueva, J., Canosa, M., Veiga, O. and Gutiérrez, A. (2011) Intestinal Perforation Due to Cutaneous Malignant Melanomametastatic Implants. Revista Española de Enfermidades Digestivas, 103, 386-388. https://doi.org/10.4321/s1130-01082011000700014

[4] Suarez, A., Ganfornina, F., Ruiz, J. and Romero, F. (2008) Intususcepción yeyunal por metástases de melanoma cutâneo. Cirugía Española, 84, 165-175. https://doi.org/10.1016/s0009-739x(08)72165-1

[5] Vaz, P., Usurelu, S., Monteiro, A., Correia, S., Gouveia, A. and Loureiro, A. (2011) Metástase de Melanoma Maligno do Intestino Delgado-Uma Rara Causa de $\mathrm{He}$ morragia Digestiva Baixa. Acta Médica Portuguesa, 24, 179-182.

[6] Patel, K., Ward, S.T., Packer, T., Brown, S., Marsden, J., Thomson, M. and Ismail, T. (2014) Malignant Melanoma of the Gastro-Intestinal Tract: A Case Series. International Journal of Surgery, 12, 523-527. https://doi.org/10.1016/j.ijsu.2014.02.011

[7] Cera, S.M. (2008) Intestinal Intussusception. Clinics in Colon and Rectal Surgery, 21, 106-113. https://doi.org/10.1055/s-2008-1075859

[8] Marsicovetere, P., Ivatury, S.J., White, B. and Holubar, S.D. (2017) Intestinal Intussusception: Etiology, Diagnosis, and Treatment. Clinics in Colon and Rectal Surgery, 30, 30-39. https://doi.org/10.1055/s-0036-1593429

[9] Agha, F.P. (1986) Intussusception in Adults. American Journal of Roentgenology, 146, 527-531.

[10] Sandrasegaran, K., Kopecky, K.K., Rajesh, A. and Lappas, J. (2004) Proximal Small Bowel Intussusceptions in Adults: CT Appearance and Clinical Significance. $A b$ dom Imaging, 29, 653-657. https://doi.org/10.1007/s00261-003-0165-0

[11] Marcoval, J., Ferreres, J.R., Martín, C., Gómez, S., Penín, R.M., Ochoa de Olza, M. and Fabra, Á. (2013) Patterns of Visceral Metastasis in Cutaneous Melanoma: A Descriptive Study. Actas Dermo-Sifiliográficas, 104, 593-597. https://doi.org/10.1016/j.ad.2012.12.012

[12] Byrne, E.H. and Fisher, D.E. (2017) Immune and Molecular Correlates in Melanoma Treated with Immune Checkpoint Blockade. Cancer, 123, 2143-2153. 
https://doi.org/10.1002/cncr.30444

[13] Namikawa, K. and Yamazaki, N. (2019) Targeted Therapy and Immunotherapy for Melanoma in Japan. Current Treatment Options in Oncology, 20, 7.

https://doi.org/10.1007/s11864-019-0607-8 